\title{
Electron-Induced Dissociation of Glycosaminoglycan Tetrasaccharides
}

\author{
Jeremy J. Wolff, ${ }^{\text {a }}$ Tatiana N. Laremore, ${ }^{\text {b }}$ Hammad Aslam, ${ }^{\text {a }}$ \\ Robert J. Linhardt, ${ }^{\mathrm{b}, c, \mathrm{~d}}$ and I. Jonathan Amster ${ }^{\mathrm{a}}$ \\ a Department of Chemistry, University of Georgia, Athens, Georgia, USA \\ ${ }^{b}$ Department of Chemistry and Chemical Biology, Rensselaer Polytechnic Institute, Troy, New York, USA \\ 'Department of Chemical and Biological Engineering, Rensselaer Polytechnic Institute, Troy, New York, USA \\ d Department of Biology, Rensselaer Polytechnic Institute, Troy, New York, USA
}

Electron detachment dissociation (EDD) Fourier transform mass spectrometry has recently been shown to be a powerful tool for examining the structural features of sulfated glycosaminoglycans (GAGs). The characteristics of GAG fragmentation by EDD include abundant cross-ring fragmentation primarily on hexuronic acid residues, cleavage of all glycosidic bonds, and the formation of even- and odd-electron product ions. GAG dissociation by EDD has been proposed to occur through the formation of an excited species that can undergo direct decomposition or ejects an electron and then undergoes dissociation. In this work, we perform electron-induced dissociation (EID) on singly charged GAGs to identify products that form via direct decomposition by eliminating the pathway of electron detachment. EID of GAG tetrasaccharides produces cleavage of all glycosidic bonds and abundant cross-ring fragmentation primarily on hexuronic acid residues, producing fragmentation similar to EDD of the same molecules, but distinctly different from the products of infrared multiphoton dissociation or collisionally activated decomposition. These results suggest that observed abundant fragmentation of hexuronic acid residues occurs as a result of their increased lability when they undergo electronic excitation. EID fragmentation of GAG tetrasaccharides results in both even- and odd-electron products. EID of heparan sulfate tetrasaccharide epimers produces identical fragmentation, in contrast to EDD, in which the epimers can be distinguished by their fragment ions. These data suggest that for EDD, electron detachment plays a significant role in distinguishing glucuronic acid from iduronic acid. (J Am Soc Mass Spectrom 2008, 19, 1449-1458) ( 2008 American Society for Mass Spectrometry

$\mathrm{G}$ lycosaminoglycans (GAGs) are complex, sulfated carbohydrates that constitute the carbohydrate portion of proteoglycans and participate in diverse biological processes, such as the regulation of biochemical pathways $[1,2]$ or inhibiting proteolysis [3], and are involved in some pathogenic infections [4-6]. GAGs are found both intra- and extracellularly in a wide variety of organisms, from bacteria to humans [7]. At the molecular level, GAGs are linear carbohydrates that consist of a repeating disaccharide containing a uronic acid (or hexose, in keratan sulfate) and a hexosamine. GAG complexity arises from variation in the degree and location of sulfation on either sugar, $\mathrm{N}$-modification of the hexosamine, and C5 stereochemistry of the uronic acid. There is significant interest in developing methods to characterize the pattern of GAG modification because these structural details are believed to influence the biological function of GAGs. A wide variety of analytical techniques have been developed to characterize sulfated GAGs, including 1D and

Address reprint requests to Dr. I. Jonathan Amster, University of Georgia, Department of Chemistry, Athens, GA 30602. E-mail: jamster@uga.edu
2D NMR [8] as well as mass spectrometry (MS) and tandem mass spectrometry (MS/MS) [9-17].

The application of electron-ion interactions to MS/MS has produced a number of useful ion dissociation techniques. The recombination of low-energy electrons $(\leq 1 \mathrm{eV})$ with multiply charged precursor ions, known as electron capture dissociation (ECD) [18], has found widespread use in the characterization of biomolecules. ECD produces a radical species that fragments differently, compared with low-energy or threshold methods of dissociation, and can be used to determine the sites of labile post-translational modifications, such as the glycosylation, sulfation, or phosphorylation of peptides and proteins [19-24]. Electron detachment dissociation (EDD) [25] is the negative-ion complement of ECD [26] and is useful for the characterization of acidic molecules that do not easily form positive ions. For EDD, multiply charged precursor anions are irradiated with moderateenergy $(\sim 19 \mathrm{eV})$ electrons, resulting in electron ejection from the precursor ion. Similar to ECD, a radical species is created that fragments differently, compared with the dissociation pathways of even-electron ions by lowenergy or threshold dissociation. 


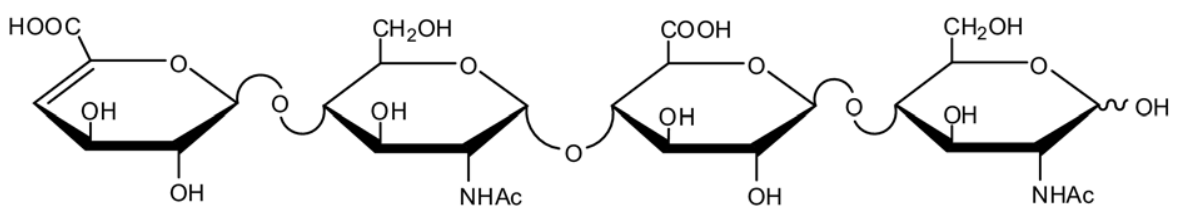

Structure 1

We have recently demonstrated the analytical utility of EDD for determining the important structural features of sulfated GAGs [27]. EDD produces more abundant cross-ring and glycosidic cleavages with minimal $\mathrm{SO}_{3}$ loss compared to collisionally activated decomposition (CAD) or infrared multiphoton dissociation (IRMPD) of the same precursor ions. EDD has been demonstrated to be useful for GAG oligosaccharides ranging in size from tetrasaccharides to decasaccharides [28] and can distinguish glucuronic acid (GlcA) from iduronic acid (IdoA) in heparan sulfate oligosaccharides by the presence of key product ions [29]. Although the experimental parameters influencing the efficiency of EDD for sulfated GAGs have recently been examined [30], the mechanistic details of GAG dissociation by EDD have not been studied. The mechanism of the EDD-induced dissociation of GAGs and the preference for GlcA/IdoA fragmentation were proposed based on the observed products [27]. Irradiation of the precursor anion with $19 \mathrm{eV}$ electrons is proposed to produce an excited intermediate that can undergo direct decomposition or electron detachment. Electron detachment from a site of negative charge produces a radical species. A preference for cross-ring fragmentation of GlcA/IdoA residues was proposed to result from radical formation at the carboxyl group, inducing fragmentation in these residues. However, recent studies on the effect of precursor ion charge on the EDD spectra show preferential fragmentation of GlcA/IdoA residues, regardless of the initial site of electron detachment, suggesting that GlcA/IdoA residues are more labile when activated via electronic excitation than by vibrational excitation [31]. Carbohydrate fragmentation by EDD has also been proposed to occur by an electroncapture mechanism [32]. Although the proposed mechanism explains the formation of even- and odd-electron products, it does not explain the preference for fragmentation of the GlcA/IdoA residues.

Some fraction of product ions in an EDD experiment is produced by activation of the precursor without detachment of an electron. The irradiation of singly charged ions with 6-20 eV electrons (i.e., below their second ionization energy but with sufficient energy to promote electronic excitation) was originally termed electron-impact excitation of ions from organics (EIEIO) $[33,34]$. It is now referred to as electron-induced dissociation (EID) [35], which also applies to the excitation of multiply charged ions with an electron beam. EID was first applied to small molecules and peptides [36], producing fragmentation similar to that of CAD or IRMPD, even though EID is expected to activate ions by electronic excitation rather than by vibrational excitation. Recent work on carbohydrate cations [35], phosphorylated metabolite anions [37], and amino acids [38] has shown promise for characterization of these small molecules using EID.

In this work, we examine the dissociation of singly charged GAG tetrasaccharides by $19 \mathrm{eV}$ electrons to distinguish products that arise from EID from those that arise from electron detachment. Because electron detachment from a singly charged GAG anion would produce a neutral molecule, products observed from irradiation of singly charged GAG precursor ions can result only from EID. The products observed from EID provide insight into the degree of fragmentation and the type of products produced by electron detachment versus EID in an EDD experiment.

\section{Experimental}

\section{Preparation of Tetrasaccharides}

Heparan sulfate tetrasaccharides 1, 2, and 4 were prepared by partial enzymatic depolymerization of heparan sulfate sodium salt (Celsus Laboratories, Cincinnati, $\mathrm{OH}$, USA). Dermatan sulfate tetrasaccharide, 3, was prepared by partial enzymatic depolymerization of porcine intestinal mucosa dermatan sulfate (Celsus Laboratories). A $20 \mathrm{mg} / \mathrm{mL}$ heparan sulfate or dermatan sulfate solution in $50 \mathrm{mM}$ Tris- $\mathrm{HCl} / 60 \mathrm{mM}$ sodium

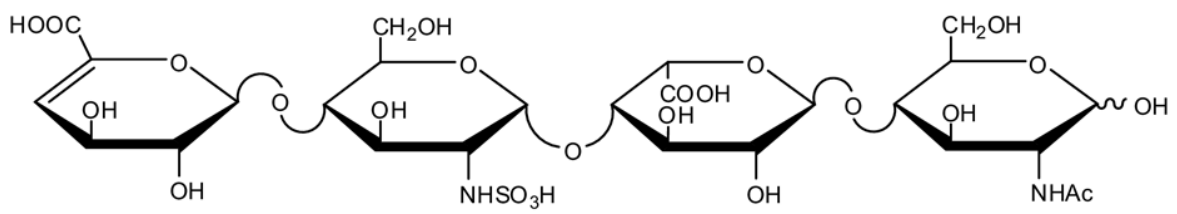

Structure 2 


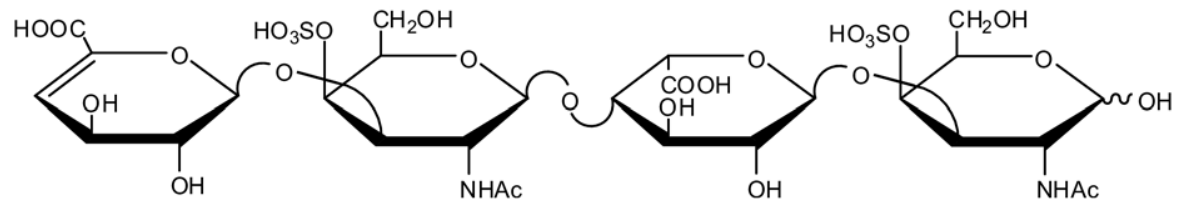

Structure 3

acetate buffer $\left(\mathrm{pH}\right.$ 8) was incubated at $37^{\circ} \mathrm{C}$ with heparinase II (Sigma, St. Louis, MO, USA) or chondroitin ABC lyase (Seikagaku Corp., Tokyo, Japan), respectively. The absorbance at $232 \mathrm{~nm}$ was monitored to determine when the digestion was $50 \%$ completed. Each digestion mixture was then heated at $100{ }^{\circ} \mathrm{C}$ for 3 min. High-molecular-weight oligosaccharides and the glycosidic enzyme were removed by ultrafiltration using a 5000 molecular weight cutoff (MWCO) membrane. The resulting oligosaccharide mixtures were each concentrated by rotary evaporation and fractionated by low-pressure gel permeation chromatography (GPC) on a Bio-Gel P10 (Bio-Rad, Richmond, CA, USA) column. Fractions containing tetrasaccharides 1, 2, 3, and 4 were desalted by GPC on a Bio-Gel P2 column and freeze-dried [39]. Further purification of the tetrasaccharides was carried out using strong anion exchange high-pressure liquid chromatography (SAXHPLC) on a semi-preparative SAX S5 Spherisorb column (Waters Corp., Milford, MA, USA). The SAXHPLC fractions containing $>90 \%$ of the tetrasaccharides were collected, desalted by GPC, and freeze-dried. The solid was reconstituted in water and purified a second time by SAX-HPLC. Only the top 30\% of the chromatographic peak was collected, desalted, and freeze-dried. Concentration of the tetrasaccharide solutions was determined by measuring the absorbance at $232 \mathrm{~nm}\left(\varepsilon=3800 \mathrm{M}^{-1} \mathrm{~cm}^{-1}\right)$. The tetrasaccharides were characterized by PAGE, electrospray ionization (ESI)MS, and high-field nuclear magnetic resonance (NMR) spectroscopy. Tetrasaccharide 5 was prepared from tetrasaccharide 2 using the following protocol: tetrasaccharide 2 sodium salt was converted to a pyridinium salt using a Dowex 50W cation exchange column (Sigma). The pyridinium salt of $2(200 \mu \mathrm{g})$ was dissolved in $10 \mu \mathrm{L}$ of dimethyl sulfoxide (Acros, Geel, Belgium) containing 5\% methanol and incubated for $1.5 \mathrm{~h}$ at $50{ }^{\circ} \mathrm{C}$ [40]. The desulfated product, 5, was then purified on a Bio-Rad P-2 spin column and freeze-dried. The product of the desulfation reaction differs in mass from that of the starting material and thus can be easily isolated from each other for the EDD experiment. The desulfation reaction does not affect the stereochemistry of the hexuronic acid residue and so the chirality of the product is the same as that of the reactant as established by NMR analysis.

\section{Mass Spectrometry Analysis}

Experiments were performed with a 9.4 T Bruker Apex Ultra QhFTMS (Billerica, MA, USA) fitted with an Apollo II dual source, a $25 \mathrm{~W} \mathrm{CO}_{2}$ laser (Synrad model J48-2, Mukilteo, WA, USA) for IRMPD, and an indirectly heated hollow cathode for generating electrons for EDD and EID. The tetrasaccharides were introduced at a concentration of $5 \mu \mathrm{M}$ in 50:50:0.1 methanol: $\mathrm{H}_{2} \mathrm{O}$ : formic acid by ESI in negative-ion mode. To produce abundant $[\mathrm{M}-\mathrm{H}]^{-}$ions for 3, the tetrasaccharide was introduced at a concentration of $50 \mu \mathrm{M}$ in $45: 45: 10$ methanol: $\mathrm{H}_{2} \mathrm{O}$ :formic acid. All sample solutions were infused at a rate of $120 \mu \mathrm{L} / \mathrm{h}$.

For the EID experiments, precursor ions were isolated in the external quadrupole and accumulated for 1-4 s before injection into the Fourier transform mass spectrometry cell. The isolation/cell fill was repeated up to six times. The selection of the precursor ion was further refined by using in-cell isolation with a coherent harmonic excitation frequency (CHEF) event [41]. The precursor ions were then irradiated with electrons for $1 \mathrm{~s}$. For electron irradiation the cathode bias was set to $-19 \mathrm{~V}$, the extraction lens was set to $-18.5 \pm 0.5 \mathrm{~V}$, and the cathode heater was set to 1.6 A. Precursor ions have been irradiated with electron energies varying from 12 to $25 \mathrm{eV}$, but no differences were observed other than changes in product ion intensity. Therefore, singly charged GAG ions were irradiated with $19 \mathrm{eV}$ electrons for comparison to previously published results. Ions were excited with a radio frequency (RF) chirp that covered the range of $100-2000 \mathrm{~m} / z$. In all, 24 acquisitions were signal averaged per mass spectrum. For each mass spectrum, $512 \mathrm{~K}$ points were acquired at a 2.4$\mathrm{MHz}$ digitization rate, padded with one zero fill, and

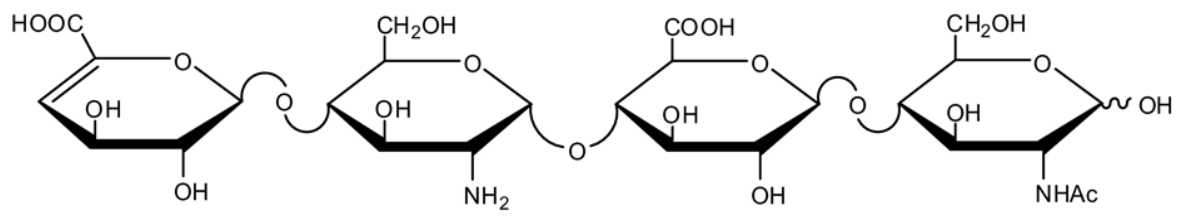

Structure 4 


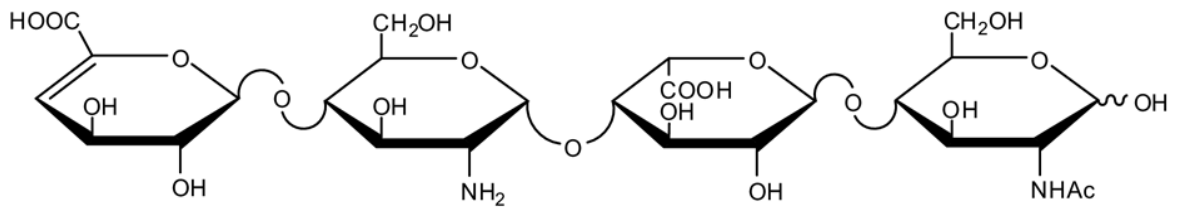

Structure 5

apodized using a sinebell window. Background spectra were acquired by leaving all parameters the same but setting the cathode bias to $0 \mathrm{~V}$, to ensure that no electrons reached the analyzer cell. IRMPD spectra were acquired using the same experimental setup as EID, but replacing the electron irradiation event with a laser pulse. For IRMPD, ions were irradiated for 0.01-0.2 s with beam attenuation set to pass from 40 to $60 \%$ of full power. External calibration of IRMPD and EID mass spectra produced mass accuracy of $5 \mathrm{ppm}$. Internal calibration was also performed using confidently assigned glycosidic bond cleavage products as internal calibrants, providing mass accuracy of $<1 \mathrm{ppm}$. Because of the larger number of low-intensity products formed by EID, only peaks with a signal-to-noise ratio $(\mathrm{S} / \mathrm{N})>10$ are reported. Product ions were assigned using accurate mass measurement. For the work presented here, fragmentation of the tetrasaccharides is presented using a modification of the Domon and Costello annotation [42] that presents GAG fragmentation with $\mathrm{SO}_{3}$ loss and hydrogen rearrangement that is observed in EDD of GAGs [28].

\section{Results and Discussion}

Irradiation of the $[\mathrm{M}-\mathrm{H}]^{-}$precursor ion of $\Delta \mathrm{UA}-$ GlcNAc-GlcA-GlcNAc, 1, with $19 \mathrm{eV}$ electrons pro- duces the EID mass spectrum shown in Figure 1a, whereas IRMPD of the same precursor ion produces the mass spectrum shown in Figure $1 \mathrm{~b}$. Although the EID mass spectrum contains all the products found by IRMPD of 1, EID of 1 also results in many additional glycosidic and cross-ring cleavages, as shown in Figure $2 \mathrm{a}$ and $\mathrm{b}$. The EDD mass spectrum of $\mathbf{1}$ has been published previously [27] and the products that result from irradiation of the $[\mathrm{M}-2 \mathrm{H}]^{2-}$ precursor ion of 1 with $19 \mathrm{eV}$ electrons (i.e., EDD of 1) are shown in Figure 2c. Interestingly, EID of the singly charged precursor ion of 1 produces identical fragmentation products to those observed by EDD of the $2^{-}$precursor, but differences in product ion intensity between EDD and EID are observed. Cleavage of all glycosidic bonds is observed and cross-ring fragmentation occurs primarily on GlcA residues. However, a few more glycosidic cleavages (the isobaric $\mathrm{C}_{2} / \mathrm{Z}_{2}$ glycosidic cleavages) are observed in EID of $\mathbf{1}$ because they do not overlap in mass-to-charge with the precursor ion as they do for the doubly charged precursor.

Cross-ring fragmentation in the amino sugar residues is found not only by IRMPD but also by EID. In contrast, only EID leads to cross-ring fragmentation in the uronic acid residues. The preference for cross-ring fragmentation in GlcA/IdoA residues has been observed in EDD of GAG tetrasaccharides to decasaccha-

(a)

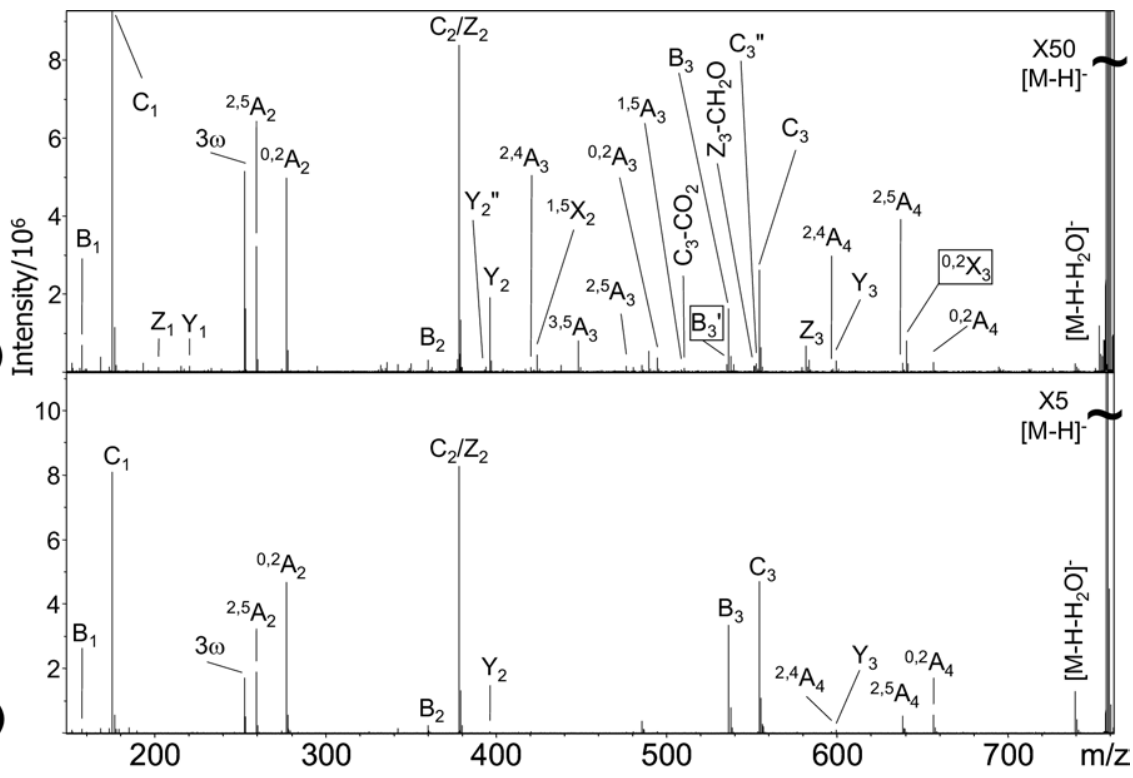

Figure 1. Tandem mass spectrum of the $[\mathrm{M}-\mathrm{H}]^{-}$precursor ion of 1 obtained by (a) EID and (b) IRMPD. Odd-electron product ions are identified with a box surrounding the peak annotation. 
(a)

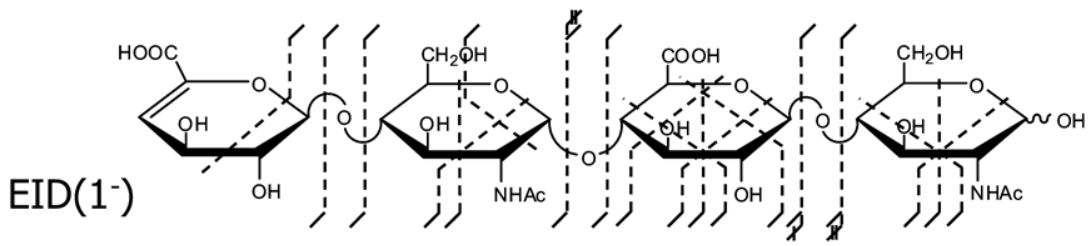

(b) $\operatorname{IRMPD}\left(1^{-}\right)$

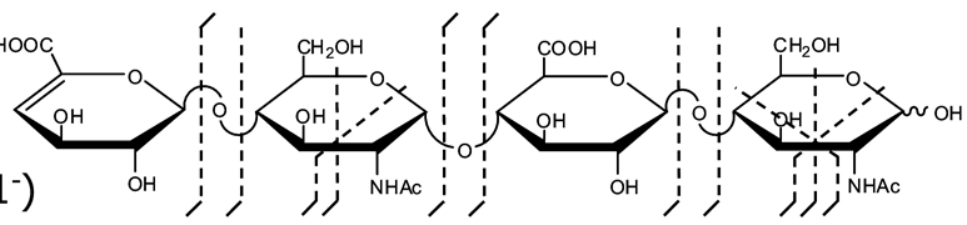

(c)

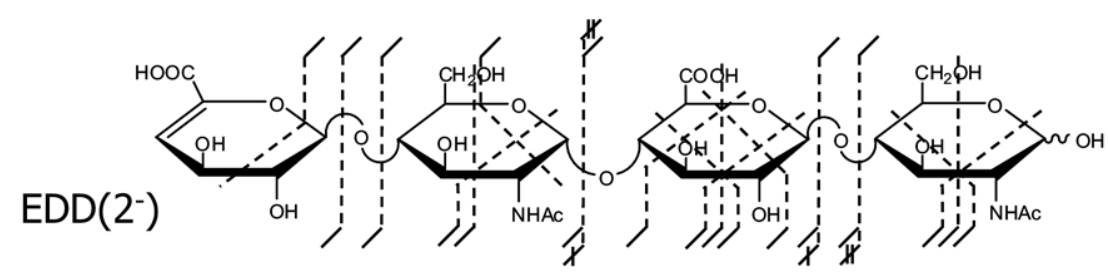

Figure 2. Products observed from (a) EID, (b) IRMPD, and (c) EDD of 1.

rides [28]. We have recently shown that GlcA/IdoA residues are particularly labile and that their lability is a result of electronic excitation rather than the presence of a radical intermediate [31]. In support of these observations, EID of $\mathbf{1}$ produces abundant cross-ring fragmentation of GlcA residues. Because these crossring cleavages are not observed by IRMPD (Figure 1b), apparently cross-ring fragmentation of GlcA occurs only by electronic excitation of the even-electron precursor ion.

EID of 1 yields some product ions that have previously been observed only in EDD mass spectra. These product ions include both even- and odd-electron ions, such as those arising from glycosidic cleavages accompanied by the loss of one or two hydrogen atoms (e.g., $\mathrm{B}_{3}{ }^{\prime}$ and $\mathrm{C}_{3}{ }^{\prime \prime}$ ) and odd-electron cross-ring cleavages (e.g., ${ }^{0,2} X_{3}$ ). Odd-electron products are indicated in Figure 2a with a box surrounding the peak annotation. For the work presented here, a box surrounding the peak annotation will identify odd-electron products observed by EID. As can be seen, the number of oddelectron products is relatively small and their abundances are low to moderate in intensity. Nevertheless, their occurrence here is surprising. Previously, we proposed that formation of these odd-electron products occurs through an odd-electron intermediate formed via electron detachment. This proposed fragmentation scheme was based on the assumption that odd-electron product ions can arise only from an odd-electron precursor ion. However, the presence of singly charged odd-electron products in EID, but not IRMPD, of $\mathbf{1}$ indicates that odd-electron products can also arise from electronic excitation of an even-electron precursor ion, albeit they are present at relatively low abundance. It is unusual for an even-electron precursor to yield an odd-electron product and relatively few instances can be found in the literature [43,44]. The EID of negatively charged metabolite ions has recently been published, but odd-electron products were not reported [37].

\section{EID of GAG Tetrasaccharides Containing Sulfate}

Irradiation of the $[\mathrm{M}-\mathrm{H}]^{-}$precursor ion of tetrasaccharide $\Delta \mathrm{UA}-\mathrm{GlcNSO}_{3}$-IdoA-GlcNAc, 2, with $19 \mathrm{eV}$ electrons produces the EID mass spectrum shown in Figure 3a, whereas IRMPD of the same precursor ion is shown in Figure 3b. The assignments of the product ions for both EID and IRMPD are shown in Figure 4a and $b$. The EDD mass spectrum of 2 has been published previously [29] and products observed from EDD of the $[\mathrm{M}-2 \mathrm{H}]^{2-}$ precursor ion of 2 are shown in Figure 4c. Irradiation of the $[\mathrm{M}-\mathrm{H}]^{-}$precursor ion of the dermatan sulfate GAG, $\triangle \mathrm{UA}$-GalNAc-4$\mathrm{SO}_{3}$-IdoA-GalNAc-4-SO $\mathrm{SO}_{3}$, 3, with $19 \mathrm{eV}$ electrons produces the EID mass spectrum shown in Figure $5 \mathrm{a}$, whereas IRMPD of the same precursor ion is shown in Figure 5b. Product ions for both EID and IRMPD are shown in Figure 6a and b. The EDD mass spectra of 3 has been published previously [28] and products observed from EDD of the $[\mathrm{M}-2 \mathrm{H}]^{2-}$ precursor ion of 3 are shown in Figure 6c.

For 2 and 3, EID produces considerably more extensive glycosidic and cross-ring fragmentation than IRMPD, as shown in Figures 4 and 6, respectively. Generally, EID produces similar fragmentation to EDD of the same molecules. However, a greater number of cross-ring cleavages are observed for EDD of these tetrasaccharides, occurring primarily across hexuronic acid residues. The additional cross-ring fragmentation products that are not observed by either EID or IRMPD, but are observed by EDD, may arise as a result of 
(a)

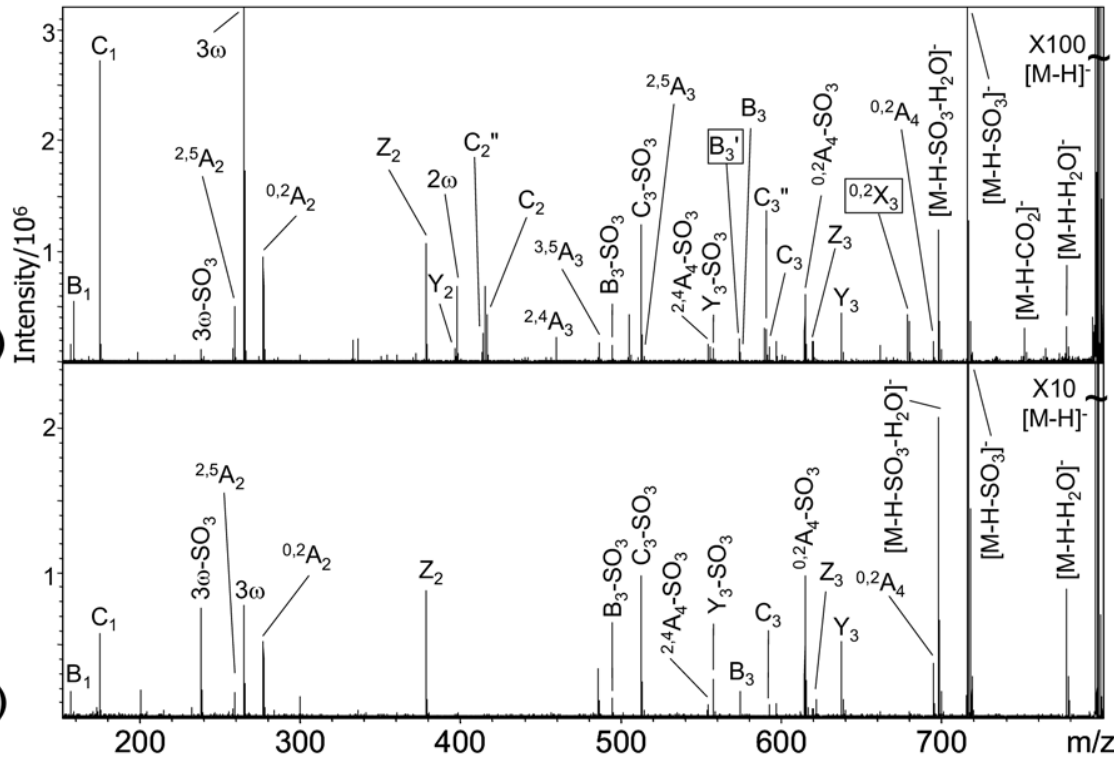

Figure 3. Tandem mass spectrum of the $\left[\mathrm{M}-\mathrm{H}^{-}\right.$precursor ion of 2 obtained by (a) EID and (b) IRMPD. Odd-electron product ions are identified with a box surrounding the peak annotation.

electron detachment from the multiply charged precursor ion. In support of the observations made on EID of 1, cross-ring cleavages occur primarily on hexuronic acid residues, indicating that these residues are most labile and readily undergo fragmentation as a result of electronic excitation. Similarly, the presence of oddelectron products, such as ${ }^{0,2} \mathrm{X}_{3}$, and glycosidic cleavages accompanied by the loss of one or two hydrogen atoms, such as $\mathrm{B}_{3}{ }^{\prime}$ and $\mathrm{C}_{3}{ }^{\prime \prime}$, are also observed in EID of 2 and 3. Cross-ring fragmentation of the reducing end residue is observed by EID of $\mathbf{1}$ and 2, but is not observed in EID of 3 , suggesting that the $1 \rightarrow 3$ glycosidic linkage inhibits this pathway of fragmentation. These observations are consistent with previous low-energy CAD studies that show that $1 \rightarrow 4$ linked HexNAc residues undergo facile retro-Diels-Alder reactions to yield ${ }^{0,2}$ A products, but not $1 \rightarrow 3$ linked HexNAc residues [45, 46]; similar results have been observed before for EDD of dermatan sulfate oligosaccharides [28] and for CAD of isobaric GAG oligosaccharides differing only in linkage position [47].

The presence of odd-electron product ions from EID suggests that different dissociation pathways are accessed by electronic activation of GAG precursor anions compared to slow-heating or low-energy ion activation. Generally speaking, odd-electron prod-

(a)

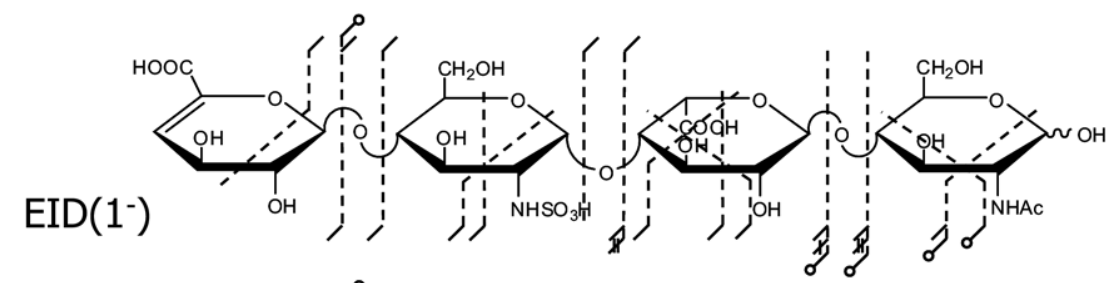

(b)

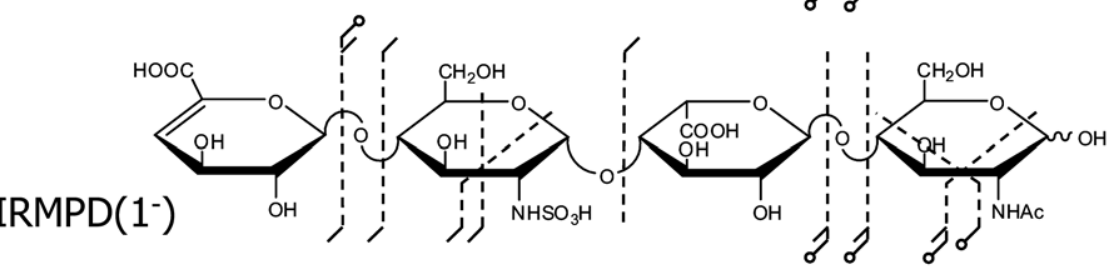

(c)

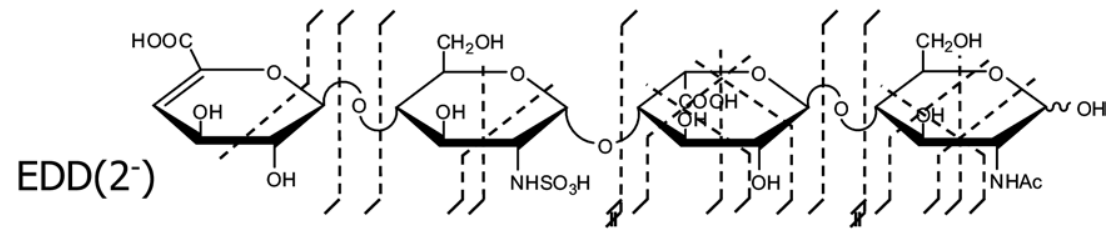

Figure 4. Products observed from (a) EID, (b) IRMPD, and (c) EDD of 2. 
(a)

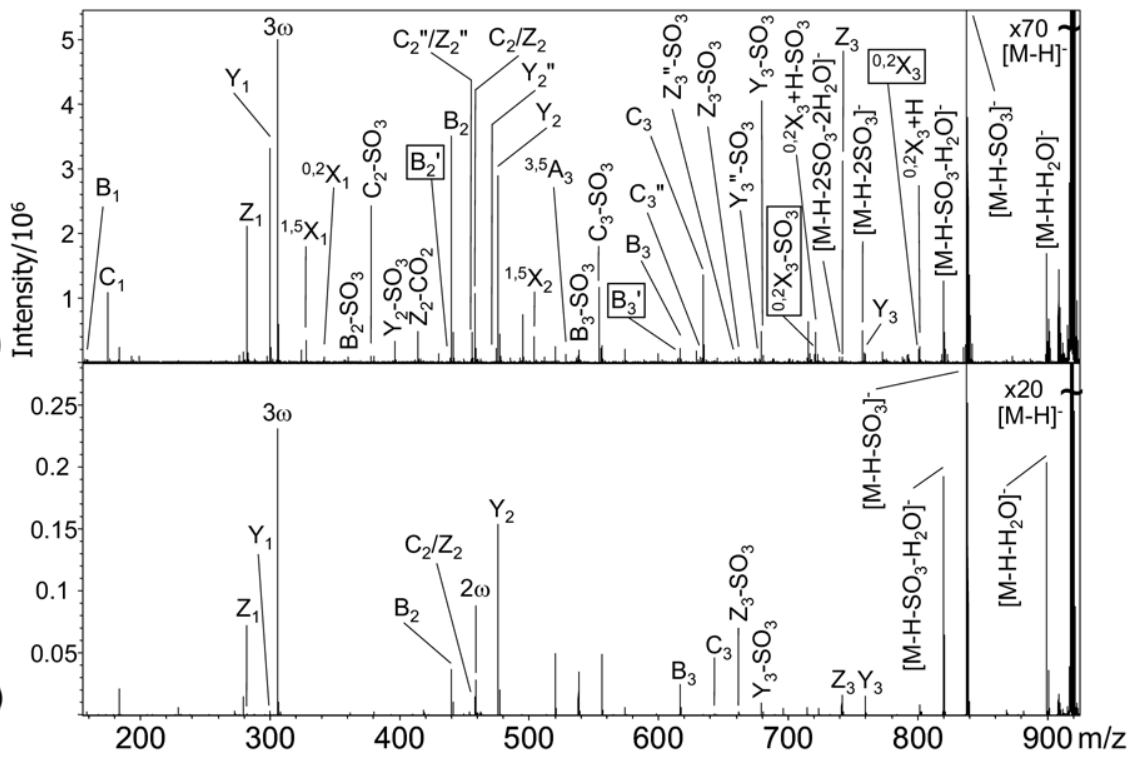

Figure 5. Tandem mass spectrum of the $[\mathrm{M}-\mathrm{H}]^{-}$precursor ion of 3 obtained by (a) EID and (b) IRMPD. Odd-electron product ions are identified with a box surrounding the peak annotation.

ucts are not produced from even-electron precursors. Their presence here raises questions about the mechanisms of dissociation. Perhaps electronic excitation produces a diradical that fragments to give a radical ion and a radical neutral. Another possibility is that electron capture produces an odd-electron doubly charged ion that fragments to a singly charged radical product ion. Although electron capture by an anion seems energetically unfavorable, direct evidence of such a process has been recently reported [32]. In the measurements made for the current study, we find no direct evidence of electron capture (i.e., no doubly charged ions were observed). These odd-electron product ions formed by EID are present over a range of electron energies studied. As shown in Figure 7, the ratio of the odd-electron $\mathrm{B}_{3}{ }^{\prime}$ to the even-electron $B_{3}$ is similar for electron energies of 19,16 , and $12 \mathrm{eV}$ (Figure 7a-c, respectively), and similar for EID of 2.

(a)

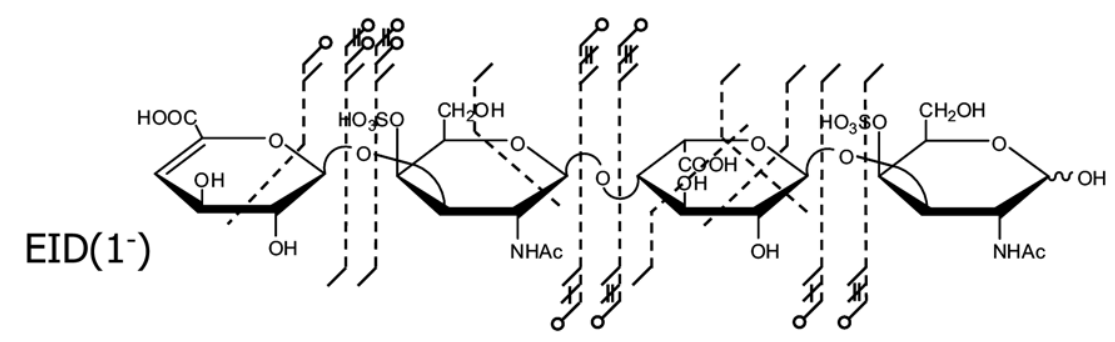

(b)

$\operatorname{IRMPD}\left(1^{-}\right)$

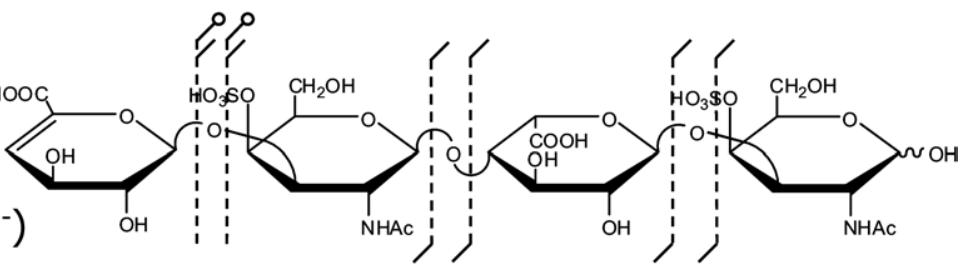

(c)

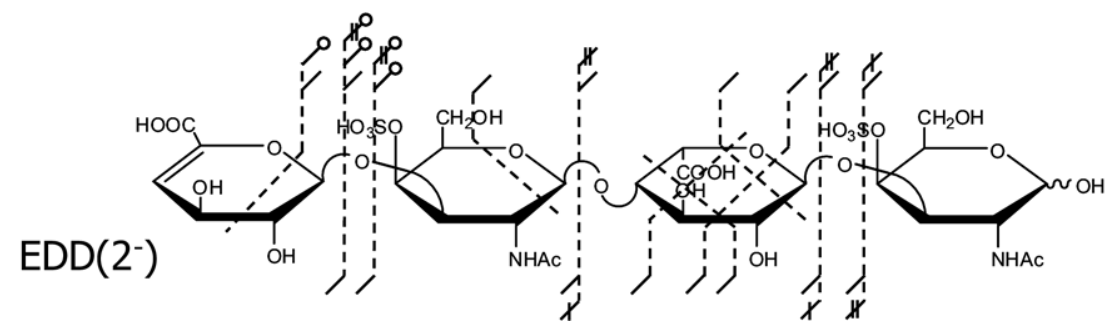

Figure 6. Products observed from (a) EID, (b) IRMPD, and (c) EDD of 3. 
(a)

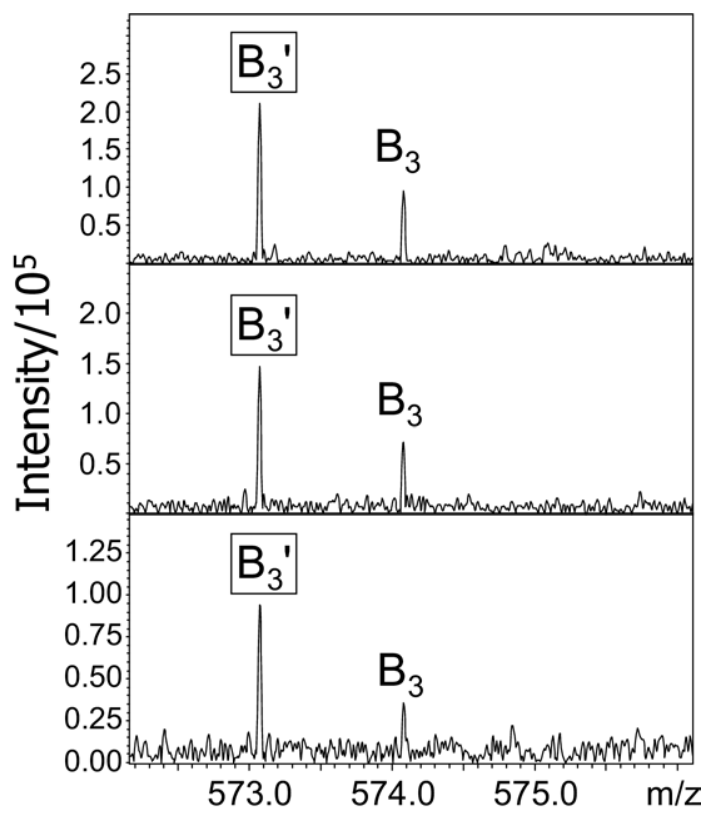

Figure 7. Ratios of the odd-electron $\mathrm{B}_{3}{ }^{\prime}$ and even-electron $\mathrm{B}_{3}$ glycosidic cleavages are similar for electron energies of (a) $19 \mathrm{eV}$, (b) $16 \mathrm{eV}$, and (c) $12 \mathrm{eV}$. The $\mathrm{S} / \mathrm{N}$ of the product ions decreases with decreasing electron energy.

However, although the ratio of the $\mathrm{B}_{3}{ }^{\prime}$ to $\mathrm{B}_{3}$ is similar for the electron energies used in this study, product ion $\mathrm{S} / \mathrm{N}$ decreases as the electron energy decreases.

Because tetrasaccharides 2 and 3 contain sulfate groups, product ions may be accompanied by the loss of $\mathrm{SO}_{3}$, which are annotated with an open circle at the end of the cleavage in Figures 4 and 6. Product ions accompanied by the loss of $\mathrm{SO}_{3}$ are observed as pairs differing by the exact mass of $\mathrm{SO}_{3}, 79.957 \mathrm{u}$, aiding in their identification. We have previously observed a significant decrease in the number of products accompanied by $\mathrm{SO}_{3}$ loss when the precursor ion charge is greater than the number of sulfate groups [31]. The reduction in $\mathrm{SO}_{3}$ loss was proposed to result from electron detachment occurring at a carboxylate anion, as opposed to electron detachment at a sulfate anion, which can then readily undergo radical-driven loss of $\mathrm{SO}_{3}$. For EID of 2 and 3, because products cannot result from electron detachment from the singly charged precursor ions, $\mathrm{SO}_{3}$ loss resulting from EID must occur via another route. There is significantly more $\mathrm{SO}_{3}$ loss observed by EID than by EDD for compound 3, primarily accompanying glycosidic bond cleavage. Previous work has shown that the abundance of ions corresponding to $\mathrm{SO}_{3}$ loss from low-energy CAD increases as the charge state of the ion decreases, suggesting that $\mathrm{SO}_{3}$ groups are most labile when associated with a proton $[48,49]$. For the singly charged GAGs in this work that have a protonated sulfate group, it is not surprising that $\mathrm{SO}_{3}$ loss is observed.

\section{EID of GAG Epimers}

We have previously reported the capability to distinguish GlcA from IdoA in GAG tetrasaccharides using EDD [29]. Product ions used to distinguish GlcA from IdoA $\left({ }^{0,2} \mathrm{~A}_{3}, \mathrm{~B}_{3}{ }^{\prime}\right.$, and $\left.\mathrm{B}_{3}{ }^{\prime}-\mathrm{CO}_{2}\right)$ were proposed to form via a radical intermediate, as a result of hydrogen atom transfer within the hexuronic acid to a carboxy radical. Because abundant cross-ring cleavages of the hexuronic acid residues are observed in EID, it is important to determine whether product ions used to distinguish GlcA from IdoA resulted from EID versus the proposed radical mechanism. EID of $\Delta$ UA-GlcNAc-GlcA-GlcNAc, 4, was compared to EID of the epimer $\Delta \mathrm{UA}$-GlcNAc-IdoA-GlcNAc, 5, shown in Figure 8a and $\mathrm{b}$, respectively. Products observed from EID of 4 and 5 are shown in the insets of Figure 8a and b, respectively. EDD mass spectra for 4 and 5 have been previously published [29]. Similar glycosidic and cross-ring fragmentation products are observed for EID of 4 and 5, as shown in the insets of Figure $8 a$ and $b$. The product ions ${ }^{0,2} \mathrm{~A}_{3}$ and $\mathrm{B}_{3}{ }^{\prime}$, used to distinguish GlcA from IdoA, are present in both EID mass spectra (Figure 8a and b) and thus do not distinguish GlcA from IdoA. Also, the $\mathrm{B}_{3}{ }^{\prime}-$ $\mathrm{CO}_{2}$ peak is not observed in either EID mass spectrum (Figure $8 \mathrm{a}$ and $\mathrm{b}$ ). These products are also not observed in IRMPD of 4 and 5 (data not shown). Clearly, the EID results are different from the results obtained by EDD of the $2^{-}$precursor ion. This suggests that either the radical species proposed for EDD [29] plays a more significant role than EID in determining the products or that the dissociation of doubly charged ions differs from that of singly charged precursors. However, no difference in glycosidic and cross-ring fragmentation is observed from IRMPD of the $1^{-}$and $2^{-}$precursor ion of 4 (data not shown), suggesting that charge state is not the primary influence in the differences between the observed product ions of EDD versus EID. Although other product ion abundances vary for the epimer pair (e.g., $B_{3}$ and $Z_{2}$ ), further work is necessary to determine whether these product ion ratios can be used to distinguish IdoA from GlcA, similar to methods demonstrated to quantitatively determine the relative amount of IdoA and GlcA by using lowenergy CAD $[12,50]$.

\section{Conclusions}

EID of singly charged GAG tetrasaccharides has been used to identify products that arise from electronic excitation. These data provide insight into the origin of products in the EDD experiment. EID of singly charged GAGs produces fragmentation products that are similar to products observed in EDD of the same molecules. EID produces cross-ring fragmentation primarily on hexuronic acid residues and both evenand odd-electron glycosidic and cross-ring products 
(a)

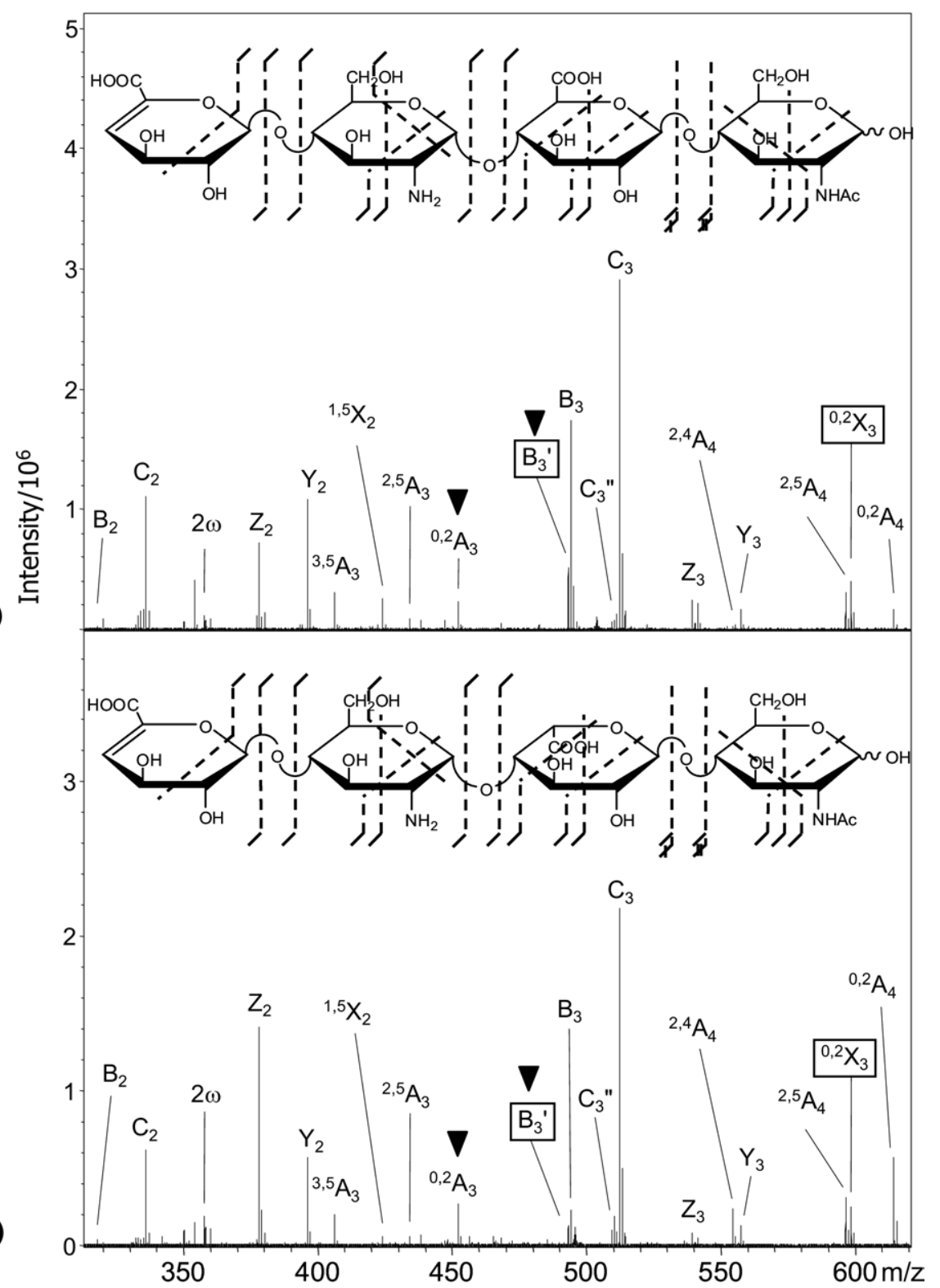

Figure 8. EID mass spectra of the epimer pair (a) 4 and (b) 5 . The mass scale is expanded to show the region containing product ions that are used to distinguish GlcA from IdoA by EDD, indicated by the $\checkmark$ over the peak label. Insets: structures indicating product ions observed from EID of (a) 4 and (b) 5. Odd-electron product ions are identified with a box surrounding the peak annotation.

are observed. These features are not observed in IRMPD of the same precursor ions. These results suggest that fragmentation of the hexuronic acid residues by EDD and EID occurs because these residues are more labile when activated by electronic excitation. Furthermore, these data show that for EDD, not all odd-electron products form via electronic detachment. However, EID mass spectra are similar for GlcA and IdoA residues in GAG epimers, in contrast to previously reported EDD results. These data suggest that a radical intermediate plays a significant role in the formation of the product ions that distinguish these epimers in EDD measurements.

\section{References}

1. Fannon, M.; Forsten, K. E.; Nugent, M. A. Potentiation and Inhibition of bFGF Binding by Heparin: A Model for Regulation of Cellular Response. Biochemistry 2000, 39, 1434-1445.

2. Wu, Z. L.; Zhang, L.; Yabe, T.; Kuberan, B.; Beeler, D. L.; Love, A.; Rosenberg, R. D. The Involvement of Heparan Sulfate (HS) in FGF1/ HS/FGFR1 Signaling Complex. J. Biol. Chem. 2003, 278, 17121-17129.

3. Sadir, R.; Imberty, A.; Baleux, F.; Lortat-Jacob, H. Heparan Sulfate/ Heparin Oligosaccharides Protect Stromal Cell-derived Factor-1 (SDF1)/CXCL12 against Proteolysis Induced by CD26/Dipeptidyl Peptidase IV. J. Biol. Chem. 2004, 279, 43854-43860.

4. Batinic, D.; Robey, F. A. The V3 Region of the Envelope Glycoprotein of Human Immunodeficiency Virus Type 1 Binds Sulfated Polysaccharides and CD4-Derived Synthetic Peptides. J. Biol. Chem. 1992, 267, 6664-6671.

5. Chen, Y.; Maguire, T.; Hileman, R. E.; Fromm, J. R.; Esko, J. D.; Linhardt, R. J.; Marks, R. M. Dengue Virus Infectivity Depends on Envelope 
Protein Binding to Target Cell Heparan Sulfate. Nat. Med. 1997, 3, 866-871.

6. Williams, R. K.; Straus, S. E. Specificity and Affinity of Binding of Herpes Simplex Virus Type 2 Glycoprotein B to Glycosaminoglycans. J. Virol. 1997, 71, 1375-1380

7. Perrimon, N.; Bernfield, M. Cellular Functions of Proteoglycans-An Overview. Semin. Cell Dev. Biol. 2001, 12, 65-67.

8. Horne, A.; Gettins, P. H. -1-NMR Spectral Assignments for 2 Series of Heparin-Derived Oligosaccharides. Carbohydr. Res. 1992, 225, 43-57.

9. Adamson, J. T.; Hakansson, K. Electron Detachment Dissociation of Neutral and Sialylated Oligosaccharides. J. Am. Soc. Mass Spectrom. 2007, 18, 2162-2172.

10. Chi, L. L.; Amster, J.; Linhardt, R. J. Mass Spectrometry for the Analysis of Highly Charged Sulfated Carbohydrates. Curr. Anal. Chem. 2005, 1, 223-240

11. Dai, Y.; Whittal, R. M.; Bridges, C. A.; Isogai, Y.; Hindsgaul, O.; Li, L. Matrix-Assisted Laser Desorption Ionization Mass Spectrometry for the Analysis of Monosulfated Oligosaccharides. Carbohydr. Res. 1997, 304, $1-9$.

12. Miller, M. J. C.; Costello, C. E.; Malmstrom, A.; Zaia, J. A Tandem Mass Spectrometric Approach to Determination of Chondroitin/Dermatan Sulfate Oligosaccharide Glycoforms. Glycobiology 2006, 16, 502-513.

13. Reinhold, V. N.; Carr, S. A.; Green, B. N.; Petitou, M.; Choay, J.; Sinay, P. Structural Characterization of Sulfated Glycosaminoglycans by FastAtom-Bombardment Mass-Spectrometry: Application to Heparin Fragments Prepared by Chemical Synthesis. Carbohydr. Res. 1987, 161, 305-313.

14. Saad, O. M.; Leary, J. A. Compositional Analysis and Quantification of Heparin and Heparan Sulfate by Electrospray Ionization Ion Trap Mass Spectrometry. Anal. Chem. 2003, 75, 2985-2995.

15. Saad, O. M.; Leary, J. A. Heparin Sequencing Using Enzymatic Digestion and ESI-MS with HOST: A Heparin/HS Oligosaccharide Sequencing Tool. Anal. Chem. 2005, 77, 5902-5911.

16. Zaia, J.; Li, X.-Q.; Chan, S. Y.; Costello, C. E. Tandem Mass Spectrometric Strategies for Determination of Sulfation Positions and Uronic Acid Epimerization in Chondroitin Sulfate Oligosaccharides. J. Am. Soc. Mass Spectrom. 2003, 14, 1270-1281.

17. Zaia, J.; McClellan, J. E.; Costello, C. E. Tandem Mass Spectrometric Determination of the 4S/6S Sulfation Sequence in Chondroitin Sulfate Oligosaccharides. Anal. Chem. 2001, 73, 6030-6039.

18. Zubarev, R. A.; Kelleher, N. L.; McLafferty, F. W. Electron Capture Dissociation of Multiply Charged Protein Cations. A Nonergodic Process. J. Am. Chem. Soc. 1998, 120, 3265-3266.

19. Hakansson, K.; Cooper, H. J.; Emmett, M. R.; Costello, C. E.; Marshall, A. G.; Nilsson, C. L. Electron Capture Dissociation and Infrared Multiphoton Dissociation MS/MS of an N-Glycosylated Tryptic Peptide to Yield Complementary Sequence Information. Anal. Chem. 2001, 73, 4530-4536.

20. Kelleher, N. L.; Zubarev, R. A.; Bush, K.; Furie, B.; Furie, B. C.; McLafferty, F. W.; Walsh, C. T. Localization of Labile Posttranslational Modifications by Electron Capture Dissociation: The Case of $\gamma$-Carboxy glutamic Acid. Anal. Chem. 1999, 71, 4250-4253.

21. Mirgorodskaya, E.; Roepstorff, P.; Zubarev, R. A. Localization of O-Glycosylation Sites in Peptides by Electron Capture Dissociation in a Fourier Transform Mass Spectrometer. Anal. Chem. 1999, 71, 4431-4436.

22. Niiranen, H.; Budnik, B. A.; Zubarev, R. A.; Auriola, S.; Lapinjoki, S. High-Performance Liquid Chromatography-Mass Spectrometry and Electron-Capture Dissociation Tandem Mass Spectrometry of Osteocalcin: Determination of Gamma-Carboxyglutamic Acid Residues. J. Chromatogr. A 2002, 962, 95-103.

23. Shi, S. D. H.; Hemling, M. E.; Carr, S. A.; Horn, D. M.; Lindh, I.; McLafferty, F. W. Phosphopeptide/Phosphoprotein Mapping by Electron Capture Dissociation Mass Spectrometry. Anal. Chem. 2001, 73, $19-22$

24. Stensballe, A.; Jensen, O. N.; Olsen, J. V.; Haselmann, K. F.; Zubarev, R. A. Electron Capture Dissociation of Singly and Multiply Phosphorylated Peptides. Rapid Commun. Mass Spectrom. 2000, 14, 1793-1800.

25. Budnik, B. A.; Haselmann, K. F.; Zubarev, R. A. Electron Detachment Dissociation of Peptide Di-Anions: An Electron-Hole Recombination Phenomenon. Chem. Phys. Lett. 2001, 342, 299-302.

26. Zubarev, R. A. Reactions of Polypeptide Ions with Electrons in the Gas Phase. Mass Spectrom. Rev. 2003, 22, 57-77.

27. Wolff, J. J.; Amster, I. J.; Chi, L.; Linhardt, R. J. Electron Detachment Dissociation of Glycosaminoglycan Tetrasaccharides. J. Am. Soc. Mass Spectrom. 2007, 18, 234-244.

28. Wolff, J. J.; Laremore, T. N.; Busch, A. M.; Linhardt, R. J.; Amster, I. J. Electron Detachment Dissociation of Dermatan Sulfate Oligosaccharides. J. Am. Soc. Mass Spectrom. 2008, 19, 294-304.

29. Wolff, J. J.; Chi, L. L.; Linhardt, R. J.; Amster, I. J. Distinguishing Glucuronic from Iduronic Acid in Glycosaminoglycan Tetrasaccharides by Using Electron Detachment Dissociation. Anal. Chem. 2007, 79, 2015-2022.

30. Leach, F. E., III; Wolff, J. J.; Laremore, T. N.; Linhardt, R. J.; Amster, I. J Evaluation of the Experimental Parameters Which Control Electron Detachment Dissociation, and Their Effect on the Fragmentation Efficiency of Glycosaminoglycan Carbohydrates. Int. J. Mass Spectrom. 2008, in press, doi:10.1016/j.ijms.2008.05.017.

31. Wolff, J. J.; Laremore, T. N.; Linhardt, R. J.; Amster, I. J. Influence of Charge State and Sodium Occupation on the Electron Detachment Dissociation and Infrared Multiphoton Dissociation of Glycosaminoglycans Oligosaccharides. I. Am. Soc. Mass Spectrom. 2008, 19, 790-798.

32. Cheng, Z.; Xie, B.; Cournoyer, J. J.; Chan, S.-Y.; Zaia, J.; Costello, C. E. O'Connor, P. B. Electron Capture Dissociation, Electron Detachment Dissociation and Collisionally Activated Dissociation Provide Complementary Structural Information Oligosaccharides, 55th ASMS Conference, Indianapolis, IN, June 3-7, 2007

33. Cody, R. B.; Freiser, B. S. Electron Impact Excitation of Ions from Organics: An Alternative to Collision Induced Dissociation. Anal. Chem. 1979, 51, 547-551.

34. Cody, R. B.; Freiser, B. S. Electron Impact Excitation of Ions in Fourier Transform Mass Spectrometry. Anal. Chem. 1987, 59, 1054-1056.

35. Budnik, B. A.; Haselmann, K. F.; Elkin, Y. N.; Gorbach, V. I.; Zubarev, R. A. Applications of Electron-Ion Dissociation Reactions for Analysis of Polycationic Chitooligosaccharides in Fourier Transform Mass Spectrometry. Anal. Chem. 2003, 75, 5994-6001.

36. Wang, B.-H.; McLafferty, F. W. Electron Impact Excitation of Ions from Larger Organic Molecules. Org. Mass Spectrom. 1990, 25, 554-556.

37. Yoo, H. J.; Liu, H.; Hakansson, K. Infrared Multiphoton Dissociation and Electron-Induced Dissociation as Alternative MS/MS Strategies for Metabolite Identification. Anal. Chem. 2007, 79, 7858-7866.

38. Lioe, H.; O'Hair, R. Comparison of Collision-Induced Dissociation and Electron-Induced Dissociation of Singly Protonated Aromatic Amino Acids, Cystine and Related Simple Peptides Using a Hybrid Linear Ion Trap-FT-ICR Mass Spectrometer. Anal. Bioanal. Chem. 2007, 389, 14291437.

39. Pervin, A.; Gallo, C.; Jandik, K. A.; Han, X. J.; Linhardt, R. J. Preparation and Structural Characterization of Large Heparin-Derived Oligosaccharides. Glycobiology 1995, 5, 83-95.

40. Inoue, Y.; Nagasawa, K. Selective N-Desulfation of Heparin with Dimethyl Sulfoxide Containing Water or Methanol. Carbohydr. Res. 1976, 46, 87-95

41. Heck, A. J. R.; de Koning, L. J.; Pinkse, F. A.; Nibbering, N. M. M. Mass-Specific Selection of Ions in Fourier-Transform Ion Cyclotron Resonance Mass Spectrometry. Unintentional Off-Resonance Cyclotron Excitation of Selected Ions. Rapid Commun. Mass Spectrom. 1991, 5, $406-414$.

42. Domon, B.; Costello, C. E. A Systematic Nomenclature for Carbohydrate Fragmentations in FAB-MS/MS Spectra of Glycoconjugates. Glycoconj. J. 1988, 5, 397-409.

43. Attygalle, A. B.; Ruzicka, J.; Varughese, D.; Bialecki, J. B.; Jafri, S Low-Energy Collision-Induced Fragmentation of Negative Ions Derived from ortho-, meta-, and para-Hydroxyphenyl Carbaldehydes, Ketones, and Related Compounds. J. Mass Spectrom. 2007, 42, 1207-1217.

44. Williams, J. P.; Nibbering, N. M. M.; Green, B. N.; Patel, V. J.; Scrivens, J. H. Collision-Induced Fragmentation Pathways Including OddElectron Ion Formation from Desorption Electrospray Ionisation Generated Protonated and Deprotonated Drugs Derived from Tandem Accurate Mass Spectrometry. J. Mass Spectrom. 2006, 41, 1277-1286.

45. Saad, O. M.; Leary, J. A. Delineating Mechanisms of Dissociation for Isomeric Heparin Disaccharides Using Isotope Labeling and Ion Trap Tandem Mass Spectrometry. J. Am. Soc. Mass Spectrom. 2004, 15, 1274-1286.

46. Spengler, B.; Dolce, J. W.; Cotter, R. J. Infrared-Laser Desorption Mass-Spectrometry of Oligosaccharides: Fragmentation Mechanisms and Isomer Analysis. Anal. Chem. 1990, 62, 1731-1737.

47. Zhang, Z.; Xie, J.; Liu, J.; Linhardt, R. J. Tandem MS Can Distinguish Hyaluronic Acid from N-Acetylheparosan. J. Am. Soc. Mass Spectrom. 2008, 19, 82-90.

48. McClellan, J. E.; Costello, C. E.; O'Connor, P. B.; Zaia, J. Influence of Charge State on Product Ion Mass Spectra and the Determination of 4S/6S Sulfation Sequence of Chondroitin Sulfate Oligosaccharides. Anal. Chem. 2002, 74, 3760-3771.

49. Naggar, E. F.; Costello, C. E.; Zaia, J. Competing Fragmentation Processes in Tandem Mass Spectrometry of Heparin-like Glycosaminoglycans. J. Am. Soc. Mass Spectrom. 2004, 15, 1534-1544.

50. Desaire, H.; Sirich, T. L.; Leary, J. A. Evidence of Block and Randomly Sequenced Chondroitin Polysaccharides: Sequential Enzymatic Digestion and Quantification Using Ion Trap Tandem Mass Spectrometry. Anal. Chem. 2001, 73, 3513-3520. 